To appear in the Journal of Applied Statistics

Vol. 00, No. 00, Month 20XX, 1-6

\title{
Supplementary material for Multiple linear regression with compositional response and covariates
}

\author{
Jiajia Chen, Xiaoqin Zhang*, Shengjia Li
}

School of Mathematical Sciences, Shanxi University, Taiyuan 030006, Shanxi, P.R. China

(Received 00 Month 20XX; accepted 00 Month 20XX)

In this supplemental material, the proofs of Theorem 3.1, Theorem 3.2, Theorem 3.3 and Theorem 3.4 are presented in Appendix A, Appendix B, Appendix C and Appendix $\mathrm{D}$, respectively.

\section{Appendix A. Proof of Theorem 3.1}

It follows from Equation (8) that

$$
\left\{\begin{array}{l}
\operatorname{ilr}\left(\boldsymbol{u}_{k i}\right)\left(\operatorname{ilr}\left(\boldsymbol{v}_{i}\right)\right)^{T}=\mathbf{W}_{D_{k}}^{T} \log \left(\boldsymbol{u}_{k i}\right)\left(\log \left(\boldsymbol{v}_{i}\right)\right)^{T} \mathbf{W}_{L}, \\
\operatorname{ilr}\left(\boldsymbol{u}_{j i}\right)\left(\operatorname{ilr}\left(\boldsymbol{u}_{k i}\right)\right)^{T}=\mathbf{W}_{D_{j}}^{T} \log \left(\boldsymbol{u}_{j i}\right)\left(\log \left(\boldsymbol{u}_{k i}\right)\right)^{T} \mathbf{W}_{D_{k}} .
\end{array}\right.
$$

Thus Equation (14) can be deduced to

$$
\begin{gathered}
\left(\begin{array}{llll}
n & \sum_{i=1}^{n}\left(\log \left(\boldsymbol{u}_{1 i}\right)\right)^{T} & \cdots & \sum_{i=1}^{n}\left(\log \left(\boldsymbol{u}_{q i}\right)\right)^{T} \\
\sum_{i=1}^{n} \log \left(\boldsymbol{u}_{1 i}\right) & \sum_{i=1}^{n} \log \left(\boldsymbol{u}_{1 i}\right)\left(\log \left(\boldsymbol{u}_{1 i}\right)\right)^{T} & \cdots & \sum_{i=1}^{n} \log \left(\boldsymbol{u}_{1 i}\right)\left(\log \left(\boldsymbol{u}_{q i}\right)\right)^{T} \\
\sum_{i=1}^{n} \log \left(\boldsymbol{u}_{2 i}\right) & \sum_{i=1}^{n} \log \left(\boldsymbol{u}_{2 i}\right)\left(\log \left(\boldsymbol{u}_{1 i}\right)\right)^{T} & \cdots & \sum_{i=1}^{n} \log \left(\boldsymbol{u}_{2 i}\right)\left(\log \left(\boldsymbol{u}_{q i}\right)\right)^{T} \\
\vdots & \vdots & \ddots \\
\sum_{i=1}^{n} \log \left(\boldsymbol{u}_{q i}\right) & \sum_{i=1}^{n} \log \left(\boldsymbol{u}_{q i}\right)\left(\log \left(\boldsymbol{u}_{1 i}\right)\right)^{T} & \cdots & \sum_{i=1}^{n} \log \left(\boldsymbol{u}_{q i}\right)\left(\log \left(\boldsymbol{u}_{q i}\right)\right)^{T}
\end{array}\right) \\
\times\left(\begin{array}{l}
\boldsymbol{b}_{0}^{T} \\
\mathbf{W}_{D_{1}} \mathbf{B}_{1}^{T} \\
\vdots \\
\mathbf{W}_{D_{q}} \mathbf{B}_{q}^{T}
\end{array}\right)=\left(\begin{array}{l}
\sum_{i=1}^{n}\left(\log \left(\boldsymbol{v}_{i}\right)\right)^{T} \mathbf{W}_{L} \\
\sum_{i=1}^{n} \log \left(\boldsymbol{u}_{1 i}\right)\left(\log \left(\boldsymbol{v}_{i}\right)\right)^{T} \mathbf{W}_{L} \\
\sum_{i=1}^{n} \log \left(\boldsymbol{u}_{2 i}\right)\left(\log \left(\boldsymbol{v}_{i}\right)\right)^{T} \mathbf{W}_{L} \\
\vdots \\
\sum_{i=1}^{n} \log \left(\boldsymbol{u}_{q i}\right)\left(\log \left(\boldsymbol{v}_{i}\right)\right)^{T} \mathbf{W}_{L}
\end{array}\right) .
\end{gathered}
$$

If both sides of Equation (A1) are multiplied from the right-hand side by matrix $\mathbf{W}_{L}^{T}$, since $\mathbf{W}_{L} \mathbf{W}_{L}^{T}=\mathbf{G}_{L}$, we can get the same solution as Equation (12), so

\footnotetext{
${ }^{*}$ Corresponding author. Email: zhangxiaoqin@sxu.edu.cn
} 


$$
\left\{\begin{array} { l } 
{ ( \operatorname { l o g } ( \boldsymbol { a } _ { 0 } ) ) ^ { T } \mathbf { G } _ { L } = \boldsymbol { b } _ { 0 } ^ { T } \mathbf { W } _ { L } ^ { T } , } \\
{ \mathbf { A } _ { j } ^ { T } = \mathbf { W } _ { D _ { j } } \mathbf { B } _ { j } ^ { T } \mathbf { W } _ { L } ^ { T } ( j = 1 , 2 , \cdots , q ) . }
\end{array} \Longrightarrow \left\{\begin{array}{l}
\boldsymbol{b}_{0}=\mathrm{ilr}\left(\boldsymbol{a}_{0}\right), \\
\mathbf{B}_{j}=\mathbf{W}_{L}^{T} \mathbf{A}_{j} \mathbf{W}_{D_{j}}(j=1,2, \cdots, q) .
\end{array}\right.\right.
$$

In fact, if we take ilr coordinates on both sides of Equation (10), according to Property 2.3 (2), we have

$$
\begin{aligned}
\operatorname{ilr}\left(\boldsymbol{v}_{i}\right) & =\operatorname{ilr}\left(\boldsymbol{a}_{0} \oplus \mathbf{A}_{1} \boxminus \boldsymbol{u}_{1 i} \oplus \cdots \oplus \mathbf{A}_{q} \boxminus \boldsymbol{u}_{q i} \oplus \varepsilon_{i}\right) \\
& =\operatorname{ilr}\left(\boldsymbol{a}_{0}\right)+\operatorname{ilr}\left(\mathbf{A}_{1} \boxminus \boldsymbol{u}_{1 i}\right)+\cdots+\operatorname{ilr}\left(\mathbf{A}_{q} \boxminus \boldsymbol{u}_{q i}\right)+\operatorname{ilr}\left(\varepsilon_{i}\right) \\
& \left.\left.=\operatorname{ilr}\left(\boldsymbol{a}_{0}\right)+\mathbf{W}_{L}^{T} \mathbf{A}_{1} \mathbf{W}_{D_{1}} \operatorname{ilr}\left(\boldsymbol{u}_{1 i}\right)\right)+\cdots+\mathbf{W}_{L}^{T} \mathbf{A}_{q} \mathbf{W}_{D_{q}} \operatorname{ilr}\left(\boldsymbol{u}_{q i}\right)\right)+\operatorname{ilr}\left(\varepsilon_{i}\right) .
\end{aligned}
$$

Corresponding to model in Equation (13), we can get the same results with Equation (A2), therefore, $\boldsymbol{a}_{0}=\operatorname{ilr}^{-1}\left(\boldsymbol{b}_{0}\right), \mathbf{A}_{j}=\mathbf{W}_{L} \mathbf{B}_{j} \mathbf{W}_{D_{j}}^{T}(j=1,2, \cdots, q)$.

\section{Appendix B. Proof of Theorem 3.2}

For any composition $\boldsymbol{v} \in \mathcal{S}^{L}$, by Equation (8), we have that

$$
\operatorname{ilr}(\boldsymbol{v})=\mathbf{W}_{L}^{T} \operatorname{clr}(\boldsymbol{v})=\mathbf{W}_{L}^{T} \operatorname{clr}\left(\mathbf{P}_{L, l_{0}}^{T} \boldsymbol{v}^{\left(l_{0}\right)}\right)=\mathbf{W}_{L}^{T} \mathbf{P}_{L, l_{0}}^{T} \operatorname{clr}\left(\boldsymbol{v}^{\left(l_{0}\right)}\right)=\mathbf{W}_{L}^{T} \mathbf{P}_{L, l_{0}}^{T} \mathbf{W}_{L} \operatorname{ilr}\left(\boldsymbol{v}^{\left(l_{0}\right)}\right),
$$

then the model in Equation (13) can be deduced to

$$
\begin{aligned}
\mathbf{W}_{L}^{T} \mathbf{P}_{L, l_{0}}^{T} \mathbf{W}_{L} \operatorname{ilr}\left(\boldsymbol{v}_{i}^{\left(l_{0}\right)}\right)= & \boldsymbol{b}_{0}+\mathbf{B}_{1} \mathbf{W}_{D_{1}}^{T} \mathbf{P}_{D_{1}, l_{1}}^{T} \mathbf{W}_{D_{1}} \operatorname{ilr}\left(\boldsymbol{u}_{1 i}^{\left(l_{1}\right)}\right) \\
& +\cdots+\mathbf{B}_{q} \mathbf{W}_{D_{q}}^{T} \mathbf{P}_{D_{q}, l_{q}}^{T} \mathbf{W}_{D_{q}} \mathrm{i} \operatorname{lr}\left(\boldsymbol{u}_{q i}^{\left(l_{q}\right)}\right)+\operatorname{ilr}\left(\varepsilon_{i}\right) .
\end{aligned}
$$

If both sides of Equation (B1) are multiplied from the left-hand side by matrix $\mathbf{W}_{L}^{T} \mathbf{P}_{L, l_{0}} \mathbf{W}_{L}$, by Property 2.1, we obtain that

$$
\begin{aligned}
\operatorname{ilr}\left(\boldsymbol{v}_{i}^{\left(l_{0}\right)}\right)= & \mathbf{W}_{L}^{T} \mathbf{P}_{L, l_{0}} \mathbf{W}_{L} \boldsymbol{b}_{0}+\mathbf{W}_{L}^{T} \mathbf{P}_{L, l_{0}} \mathbf{W}_{L} \mathbf{B}_{1} \mathbf{W}_{D_{1}}^{T} \mathbf{P}_{D_{1}, l_{1}}^{T} \mathbf{W}_{D_{1}} \operatorname{ilr}\left(\boldsymbol{u}_{1 i}^{\left(l_{1}\right)}\right)+\cdots \\
& +\mathbf{W}_{L}^{T} \mathbf{P}_{L, l_{0}} \mathbf{W}_{L} \mathbf{B}_{q} \mathbf{W}_{D_{q}}^{T} \mathbf{P}_{D_{q}, l_{q}}^{T} \mathbf{W}_{D_{q}} \operatorname{ilr}\left(\boldsymbol{u}_{q i}^{\left(l_{q}\right)}\right)+\mathbf{W}_{L}^{T} \mathbf{P}_{L, l_{0}} \mathbf{W}_{L} \operatorname{ilr}\left(\varepsilon_{i}\right)
\end{aligned}
$$

Compared with model in Equation (15), it is clear that $\boldsymbol{b}_{0}^{\left(l_{0}\right)}=\mathbf{W}_{L}^{T} \mathbf{P}_{L, l_{0}} \mathbf{W}_{L} \boldsymbol{b}_{0}, \mathbf{B}_{j}^{\left(l_{0}, l_{j}\right)}=$ $\mathbf{W}_{L}^{T} \mathbf{P}_{L, l_{0}} \mathbf{W}_{L} \mathbf{B}_{j} \mathbf{W}_{D_{j}}^{T} \mathbf{P}_{D_{j}, l_{j}}^{T} \mathbf{W}_{D_{j}}(j=1,2, \cdots, q)$.

\section{Appendix C. Proof of Theorem 3.3}

Without loss of generality, suppose that $l_{0}=l_{1}=\cdots=l_{q}=1$, the model in Equation (17) is simplified to $E(\mathbf{Y} \mid \mathbf{Z})=\mathbf{Z} \boldsymbol{\beta}$. When composition $\boldsymbol{u}_{j}(j=1,2, \cdots, q)$ is permuted through a $D_{j} \times D_{j}$ permutation matrix $\mathbf{P}_{j}$, we get the regression equation

$$
E\left(\mathbf{Y} \mid \mathbf{Z}^{(\mathbf{P})}\right)=\mathbf{Z}^{(\mathbf{P})} \boldsymbol{\beta}^{(\mathbf{P})},
$$


where $\mathbf{Z}^{(\mathbf{P})}=\left(\boldsymbol{z}_{1}^{(\mathbf{P})}, \boldsymbol{z}_{2}^{(\mathbf{P})}, \cdots, \boldsymbol{z}_{n}^{(\mathbf{P})}\right)^{T}, \boldsymbol{z}_{i}^{(\mathbf{P})}=\left(1,\left(\operatorname{ilr}\left(\mathbf{P}_{1} \boldsymbol{u}_{1 i}\right)\right)^{T},\left(\operatorname{ilr}\left(\mathbf{P}_{2} \boldsymbol{u}_{2 i}\right)\right)^{T}, \cdots\right.$, $\left.\left(\operatorname{ilr}\left(\mathbf{P}_{q} \boldsymbol{u}_{q i}\right)\right)^{T}\right)^{T}, \boldsymbol{\beta}^{(\mathbf{P})}$ is the regression coefficient vector. By Equation (8), we have

$$
\operatorname{ilr}\left(\mathbf{P}_{j} \boldsymbol{u}_{j i}\right)=\mathbf{W}_{D_{j}}^{T} \operatorname{clr}\left(\mathbf{P}_{j} \boldsymbol{u}_{j i}\right)=\mathbf{W}_{D_{j}}^{T} \mathbf{P}_{j} \operatorname{clr}\left(\boldsymbol{u}_{j i}\right)=\mathbf{W}_{D_{j}}^{T} \mathbf{P}_{j} \mathbf{W}_{D_{j}} \operatorname{llr}\left(\boldsymbol{u}_{j i}\right),
$$

Denote a $(D+1) \times(D+1)$ matrix

$$
\mathbf{P}=\left(\begin{array}{ccccc}
1 & \mathbf{0}_{D_{1}-1}^{T} & \mathbf{0}_{D_{2}-1}^{T} & \cdots & \mathbf{0}_{D_{q}-1}^{T} \\
\mathbf{0}_{D_{1}-1} & \mathbf{W}_{D_{1}}^{T} \mathbf{P}_{1} \mathbf{W}_{D_{1}} & \mathbf{0}_{\left(D_{1}-1\right) \times\left(D_{2}-1\right)} & \cdots & \mathbf{0}_{\left(D_{1}-1\right) \times\left(D_{q}-1\right)} \\
\mathbf{0}_{D_{2}-1} & \mathbf{0}_{\left(D_{2}-1\right) \times\left(D_{1}-1\right)} & \mathbf{W}_{D_{2}}^{T} \mathbf{P}_{2} \mathbf{W}_{D_{2}} & \cdots & \mathbf{0}_{\left(D_{2}-1\right) \times\left(D_{q}-1\right)} \\
\vdots & \vdots & \vdots & \ddots & \vdots \\
\mathbf{0}_{D_{q}-1} & \mathbf{0}_{\left(D_{q}-1\right) \times\left(D_{1}-1\right)} & \mathbf{0}_{\left(D_{q}-1\right) \times\left(D_{2}-1\right)} & \cdots & \mathbf{W}_{D_{q}}^{T} \mathbf{P}_{q} \mathbf{W}_{D_{q}}
\end{array}\right) .
$$

According to Property 2.1 (2), $\mathbf{P}$ is an orthogonal matrix. It is clear that we can get the following equations

$$
\left\{\begin{array}{l}
\boldsymbol{z}_{i}^{(\mathbf{P})}=\mathbf{P} \boldsymbol{z}_{i}, \\
\mathbf{Z}^{(\mathbf{P})}=\mathbf{Z} \mathbf{P}^{T}, \\
\left(\left(\mathbf{Z}^{(\mathbf{P})}\right)^{T} \mathbf{Z}^{(\mathbf{P})}\right)^{-1}=\left(\left(\mathbf{Z} \mathbf{P}^{T}\right)^{T} \mathbf{Z} \mathbf{P}^{T}\right)^{-1}=\left(\mathbf{P}^{T}\right)^{-1}\left(\mathbf{Z}^{T} \mathbf{Z}\right)^{-1} \mathbf{P}^{-1}=\mathbf{P}\left(\mathbf{Z}^{T} \mathbf{Z}\right)^{-1} \mathbf{P}^{T}, \\
\widehat{\boldsymbol{\beta}}^{(\mathbf{P})}=\left(\left(\mathbf{Z}^{(\mathbf{P})}\right)^{T} \mathbf{Z}^{(\mathbf{P})}\right)^{-1}\left(\mathbf{Z}^{(\mathbf{P})}\right)^{T} \mathbf{Y}=\mathbf{P}\left(\mathbf{Z}^{T} \mathbf{Z}\right)^{-1} \mathbf{P}^{T} \mathbf{P} \mathbf{Z}^{T} \mathbf{Y}=\mathbf{P}\left(\mathbf{Z}^{T} \mathbf{Z}\right)^{-1} \mathbf{Z}^{T} \mathbf{Y}=\mathbf{P} \widehat{\boldsymbol{\beta}}, \\
\left(S^{(\mathbf{P})}\right)^{2}=\frac{\left(\mathbf{Y}-\mathbf{Z}^{(\mathbf{P})} \widehat{\boldsymbol{\beta}}^{(\mathbf{P})}\right)^{T}\left(\mathbf{Y}-\mathbf{Z}^{(\mathbf{P})} \widehat{\boldsymbol{\beta}}^{(\mathbf{P})}\right)}{n-D-1}=\frac{\left(\mathbf{Y}-\mathbf{Z P}^{T} \mathbf{P} \widehat{\boldsymbol{\beta}}\right)^{T}\left(\mathbf{Y}-\mathbf{Z} \mathbf{P}^{T} \mathbf{P} \widehat{\boldsymbol{\beta}}\right)}{n-D-1}=S^{2},
\end{array}\right.
$$

where $\left(S^{(\mathbf{P})}\right)^{2}$ denotes the unbiased estimator of the residual variance in Equation (C1).

(1) Without loss of generality, suppose that $j=1$. If the remaining components of $\boldsymbol{u}_{1}$ except for the first component are permuted through permutation matrix $\mathbf{Q}_{1}$, then

$$
\begin{aligned}
& \mathbf{W}_{D_{1}}^{T} \mathbf{P}_{1} \mathbf{W}_{D_{1}} \\
& =\left(\begin{array}{cc}
\sqrt{\frac{D_{1}-1}{D_{1}}} & -\frac{1}{\sqrt{D_{1}\left(D_{1}-1\right)}} \boldsymbol{j}_{D_{1}-1}^{T} \\
\mathbf{0}_{D_{1}-2} & \mathbf{W}_{D_{1}-1}^{T}
\end{array}\right)\left(\begin{array}{cc}
1 & \mathbf{0}_{D_{1}-1}^{T} \\
\mathbf{0}_{D_{1}-1} & \mathbf{Q}_{1}
\end{array}\right)\left(\begin{array}{cc}
\sqrt{\frac{D_{1}-1}{D_{1}}} & \mathbf{0}_{D_{1}-2}^{T} \\
-\frac{1}{\sqrt{D_{1}\left(D_{1}-1\right)}} \boldsymbol{j}_{D_{1}-1} & \mathbf{W}_{D_{1}-1}
\end{array}\right) \\
& =\left(\begin{array}{cc}
\frac{D_{1}-1}{D_{1}}+\frac{1}{D_{1}\left(D_{1}-1\right)} \boldsymbol{j}_{D_{1}-1}^{T} \mathbf{Q}_{1} \boldsymbol{j}_{D_{1}-1} & -\frac{1}{\sqrt{D_{1}\left(D_{1}-1\right)}} \boldsymbol{j}_{D_{1}-1}^{T} \mathbf{Q}_{1} \mathbf{W}_{D_{1}-1} \\
-\frac{1}{\sqrt{D_{1}\left(D_{1}-1\right)}} \mathbf{W}_{D_{1}-1}^{T} \mathbf{Q}_{1} \boldsymbol{j}_{D_{1}-1} & \mathbf{W}_{D_{1}-1}^{T} \mathbf{Q}_{1} \mathbf{W}_{D_{1}-1}
\end{array}\right) \\
& =\left(\begin{array}{cc}
1 & \mathbf{0}_{D_{1}-2}^{T}-2 \\
\mathbf{0}_{D_{1}-2} & \mathbf{W}_{D_{1}-1}^{T} \mathbf{Q}_{1} \mathbf{W}_{D_{1}-1}
\end{array}\right) .
\end{aligned}
$$

If the components of $\boldsymbol{u}_{k}(k \neq 1)$ are permuted through arbitrary permutation matrix $\mathbf{P}_{k}(k \neq 1)$, then the permutation matrix $\mathbf{P}$ in Equation (C2) can be expressed as

$$
\mathbf{P}=\left(\begin{array}{cccccc}
1 & 0 & \mathbf{0}_{D_{1}-2}^{T} & \mathbf{0}_{D_{2}-1}^{T} & \cdots & \mathbf{0}_{D_{q}-1}^{T} \\
0 & 1 & \mathbf{0}_{D_{1}-2}^{T} & \mathbf{0}_{D_{2}-1}^{T} & \cdots & \mathbf{0}_{D_{q}-1}^{T} \\
\mathbf{0}_{D_{1}-2} & \mathbf{0}_{D_{1}-2} & \mathbf{W}_{D_{1}-1}^{T} \mathbf{Q}_{1} \mathbf{W}_{D_{1}-1} & \mathbf{0}_{\left(D_{1}-2\right) \times\left(D_{2}-1\right)} & \cdots & \mathbf{0}_{\left(D_{1}-2\right) \times\left(D_{q}-1\right)} \\
\mathbf{0}_{D_{2}-1} & \mathbf{0}_{D_{2}-1} & \mathbf{0}_{\left(D_{2}-1\right) \times\left(D_{1}-2\right)} & \mathbf{W}_{D_{2}}^{T} \mathbf{P}_{2} \mathbf{W}_{D_{2}} & \cdots & \mathbf{0}_{\left(D_{2}-1\right) \times\left(D_{q}-1\right)} \\
\vdots & \vdots & \vdots & \vdots & \ddots & \vdots \\
\mathbf{0}_{D_{q}-1} & \mathbf{0}_{D_{q}-1} & \mathbf{0}_{\left(D_{q}-1\right) \times\left(D_{1}-2\right)} & \mathbf{0}_{\left(D_{q}-1\right) \times\left(D_{2}-1\right)} & \cdots & \mathbf{W}_{D_{q}}^{T} \mathbf{P}_{q} \mathbf{W}_{D_{q}}
\end{array}\right)
$$


Put permutation matrix $\mathbf{P}$ in Equation (C5) into Equation (C3), we get $\beta_{0}=$ $\beta_{0}^{(\mathbf{P})}, \beta_{1}=\beta_{1}^{(\mathbf{P})}$, and

$$
\begin{aligned}
& \left\{\left(\left(\mathbf{Z}^{(\mathbf{P})}\right)^{T} \mathbf{Z}^{(\mathbf{P})}\right)^{-1}\right\}_{1,1}=\boldsymbol{e}_{D+1,1}^{T}\left(\left(\mathbf{Z}^{(\mathbf{P})}\right)^{T} \mathbf{Z}^{(\mathbf{P})}\right)^{-1} \boldsymbol{e}_{D+1,1}=\boldsymbol{e}_{D+1,1}^{T} \mathbf{P}\left(\mathbf{Z}^{T} \mathbf{Z}\right)^{-1} \mathbf{P}^{T} \boldsymbol{e}_{D+1,1} \\
= & \boldsymbol{e}_{D+1,1}^{T}\left(\mathbf{Z}^{T} \mathbf{Z}\right)^{-1} \boldsymbol{e}_{D+1,1}=\left\{\left(\mathbf{Z}^{T} \mathbf{Z}\right)^{-1}\right\}_{1,1}, \\
& \left\{\left(\left(\mathbf{Z}^{(\mathbf{P})}\right)^{T} \mathbf{Z}^{(\mathbf{P})}\right)^{-1}\right\}_{2,2}=\boldsymbol{e}_{D+1,2}^{T}\left(\left(\mathbf{Z}^{(\mathbf{P})}\right)^{T} \mathbf{Z}^{(\mathbf{P})}\right)^{-1} \boldsymbol{e}_{D+1,2}=\boldsymbol{e}_{D+1,2}^{T} \mathbf{P}\left(\mathbf{Z}^{T} \mathbf{Z}\right)^{-1} \mathbf{P}^{T} \boldsymbol{e}_{D+1,2} \\
= & \boldsymbol{e}_{D+1,2}^{T}\left(\mathbf{Z}^{T} \mathbf{Z}\right)^{-1} \boldsymbol{e}_{D+1,2}=\left\{\left(\mathbf{Z}^{T} \mathbf{Z}\right)^{-1}\right\}_{2,2} .
\end{aligned}
$$

Since $\left(S^{(\mathbf{P})}\right)^{2}=S^{2}$, the test statistics $T_{0}$ and $T_{1}$ are invariant.

(2) If the remaining components of $\boldsymbol{u}_{j}(j=1,2, \cdots, q)$ except for the first component are permuted through permutation matrix $\mathbf{Q}_{j}$, similar to Equation (C4), then the permutation matrix $\mathbf{P}$ in Equation $(\mathrm{C} 2)$ can be expressed as:

$$
\mathbf{P}=\left(\begin{array}{cccccc}
1 & 0 & \mathbf{0}_{D_{1}-2}^{T} & \cdots & 0 & \mathbf{0}_{D_{q}-2}^{T} \\
0 & 1 & \mathbf{0}_{D_{1}-2}^{T} & \cdots & 0 & \mathbf{0}_{D_{q}-2}^{T} \\
\mathbf{0}_{D_{1}-2} & \mathbf{0}_{D_{1}-2} & \mathbf{W}_{D_{1}-1}^{T} \mathbf{Q}_{1} \mathbf{W}_{D_{1}-1} & \cdots & \mathbf{0}_{D_{1}-2} & \mathbf{0}_{\left(D_{1}-2\right) \times\left(D_{q}-2\right)} \\
\vdots & \vdots & \vdots & \ddots & \vdots & \vdots \\
0 & 0 & \mathbf{0}_{D_{1}-2}^{T} & \cdots & 1 & \mathbf{0}_{D_{q}-2}^{T} \\
\mathbf{0}_{D_{q}-2} & \mathbf{0}_{D_{q}-2} & \mathbf{0}_{\left(D_{q}-2\right) \times\left(D_{1}-2\right)} & \cdots & \mathbf{0}_{D_{q}-2} & \mathbf{W}_{D_{q}-1}^{T} \mathbf{Q}_{q} \mathbf{W}_{D_{q}-1}
\end{array}\right)
$$

It follows from Equation (C3) and Equation (C6) that the parameters $\beta_{0}, \beta_{k_{j}}(j=$ $1,2, \cdots, q)$ remain unchanged, and

$$
\begin{gathered}
\left\{\left(\left(\mathbf{Z}^{(\mathbf{P})}\right)^{T} \mathbf{Z}^{(\mathbf{P})}\right)^{-1}\right\}_{1,1}=\boldsymbol{e}_{D+1,1}^{T} \mathbf{P}\left(\mathbf{Z}^{T} \mathbf{Z}\right)^{-1} \mathbf{P}^{T} \boldsymbol{e}_{D+1,1}=\left\{\left(\mathbf{Z}^{T} \mathbf{Z}\right)^{-1}\right\}_{1,1}, \\
\\
\quad\left\{\left(\left(\mathbf{Z}^{(\mathbf{P})}\right)^{T} \mathbf{Z}^{(\mathbf{P})}\right)^{-1}\right\}_{k_{j}+1, k_{j}+1}=\boldsymbol{e}_{D+1, k_{j}+1}^{T} \mathbf{P}\left(\mathbf{Z}^{T} \mathbf{Z}\right)^{-1} \mathbf{P}^{T} \boldsymbol{e}_{D+1, k_{j}+1} \\
=\boldsymbol{e}_{D+1, k_{j}+1}^{T}\left(\mathbf{Z}^{T} \mathbf{Z}\right)^{-1} \boldsymbol{e}_{D+1, k_{j}+1}=\left\{\left(\mathbf{Z}^{T} \mathbf{Z}\right)^{-1}\right\}_{k_{j}+1, k_{j}+1},
\end{gathered}
$$

where $k_{j}=\sum_{i=1}^{j} D_{i}-D_{j}-j+2(j=1, \cdots, q)$. Since $\left(S^{(\mathbf{P})}\right)^{2}=S^{2}$, the test statistics $T_{0}$ and $T_{k_{j}}(j=1,2, \cdots, q)$ are invariant.

(3) If the components of $\boldsymbol{u}_{j}(j=1,2, \cdots, q)$ are permuted through permutation matrix $\mathbf{P}_{j}$, for convenience, the permutation matrix $\mathbf{P}$ in Equation (C2) is denoted as

$$
\mathbf{P}=\left(\begin{array}{cc}
1 & \mathbf{0}_{D}^{T} \\
\mathbf{0}_{D} & \mathbf{Q}
\end{array}\right)
$$

where $\mathbf{Q}$ is a permutation matrix. Put permutation matrix $\mathbf{P}$ in Equation $(\mathrm{C} 7)$ into Equation (C3), it is obvious that the test statistic $T_{0}$ is invariant. The test statistic 
for testing whether all the parameters in Equation $(\mathrm{C} 1)$ are equal to 0 is

$$
\begin{aligned}
& \frac{1}{D\left(S^{(\mathbf{P})}\right)^{2}}\left(\widehat{\boldsymbol{\beta}}_{*}^{(\mathbf{P})}\right)^{T}\left\{\left(\left(\mathbf{Z}^{(\mathbf{P})}\right)^{T} \mathbf{Z}^{(\mathbf{P})}\right)^{-1}\right\}_{-1,-1} \widehat{\boldsymbol{\beta}}_{*}^{(\mathbf{P})} \\
& =\frac{1}{D\left(S^{(\mathbf{P})}\right)^{2}}\left(\left(\mathbf{0}_{D}: \mathbf{I}_{D}\right) \widehat{\boldsymbol{\beta}}^{(\mathbf{P})}\right)^{T}\left(\mathbf{0}_{D}: \mathbf{I}_{D}\right)\left(\left(\mathbf{P}^{(\mathbf{P})}\right)^{T} \mathbf{Z}^{(\mathbf{P})}\right)^{-1}\left(\mathbf{0}_{D}: \mathbf{I}_{D}\right)^{T}\left(\mathbf{0}_{D}: \mathbf{I}_{D}\right) \widehat{\boldsymbol{\beta}}^{(\mathbf{P})} \\
& =\frac{1}{D\left(S^{(\mathbf{P})}\right)^{2}}\left(\widehat{\boldsymbol{\beta}}^{(\mathbf{P})}\right)^{T}\left(\begin{array}{cc}
0 & \mathbf{0}_{D}^{T} \\
\mathbf{0}_{D} & \mathbf{I}_{D}
\end{array}\right)\left(\left(\mathbf{Z}^{(\mathbf{P})}\right)^{T} \mathbf{Z}^{(\mathbf{P})}\right)^{-1}\left(\begin{array}{cc}
0 & \mathbf{0}_{D}^{T} \\
\mathbf{0}_{D} & \mathbf{I}_{D}
\end{array}\right) \widehat{\boldsymbol{\beta}}^{(\mathbf{P})}
\end{aligned}
$$

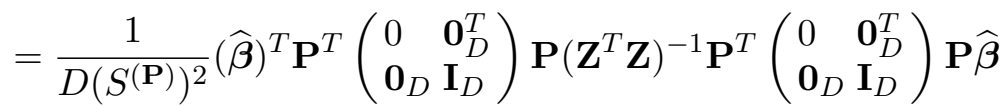

$$
\begin{aligned}
& =\frac{1}{D\left(S^{(\mathbf{P})}\right)^{2}}(\widehat{\boldsymbol{\beta}})^{T}\left(\begin{array}{cc}
0 & \mathbf{0}_{D}^{T} \\
\mathbf{0}_{D} & \mathbf{I}_{D}
\end{array}\right)\left(\mathbf{Z}^{T} \mathbf{Z}\right)^{-1}\left(\begin{array}{cc}
0 & \mathbf{0}_{D}^{T} \\
\mathbf{0}_{D} & \mathbf{I}_{D}
\end{array}\right) \widehat{\boldsymbol{\beta}} \\
& =\frac{1}{D\left(S^{(\mathbf{P})}\right)^{2}}\left(\left(\mathbf{0}_{D}: \mathbf{I}_{D}\right) \widehat{\boldsymbol{\beta}}\right)^{T}\left(\mathbf{0}_{D}: \mathbf{I}_{D}\right)\left(\mathbf{Z}^{T} \mathbf{Z}\right)^{-1}\left(\mathbf{0}_{D}: \mathbf{I}_{D}\right)^{T}\left(\mathbf{0}_{D}: \mathbf{I}_{D}\right) \widehat{\boldsymbol{\beta}} \\
& =\frac{1}{D S^{2}}\left(\widehat{\boldsymbol{\beta}}_{*}\right)^{T}\left\{\left(\mathbf{Z}^{T} \mathbf{Z}\right)^{-1}\right\}_{-1,-1} \widehat{\boldsymbol{\beta}}_{*},
\end{aligned}
$$

so the test statistic $F$ is invariant under the permutation of $\boldsymbol{u}_{j}$.

\section{Appendix D. Proof of Theorem 3.4}

According to Property 2.1, Theorem 3.1 and Theorem 3.2, we obtain that

$$
\begin{aligned}
\boldsymbol{c}_{0} & =\left(\begin{array}{c}
\boldsymbol{e}_{L-1,1}^{T} \boldsymbol{b}_{0}^{(1)} \\
\boldsymbol{e}_{L-1,1}^{T} \boldsymbol{b}_{0}^{(2)} \\
\vdots \\
\boldsymbol{e}_{L-1,1}^{T} \boldsymbol{b}_{0}^{(L)}
\end{array}\right)=\left(\begin{array}{c}
\boldsymbol{e}_{L-1,1}^{T} \mathbf{W}_{L}^{T} \mathbf{P}_{L, 1} \mathbf{W}_{L} \boldsymbol{b}_{0} \\
\boldsymbol{e}_{L-1,1}^{T} \mathbf{W}_{L}^{T} \mathbf{P}_{L, 2} \mathbf{W}_{L} \boldsymbol{b}_{0} \\
\vdots \\
\boldsymbol{e}_{L-1,1}^{T} \mathbf{W}_{L}^{T} \mathbf{P}_{L, L} \mathbf{W}_{L} \boldsymbol{b}_{0}
\end{array}\right)=\left(\begin{array}{c}
\boldsymbol{e}_{L-1,1}^{T} \mathbf{W}_{L}^{T} \mathbf{P}_{L, 1} \\
\boldsymbol{e}_{L-1,1}^{T} \mathbf{W}_{L}^{T} \mathbf{P}_{L, 2} \\
\vdots \\
\boldsymbol{e}_{L-1,1}^{T} \mathbf{W}_{L}^{T} \mathbf{P}_{L, L}
\end{array}\right) \mathbf{W}_{L} \boldsymbol{b}_{0} \\
& =\sqrt{\frac{L}{L-1}} \mathbf{G}_{L} \mathbf{W}_{L} \boldsymbol{b}_{0}=\sqrt{\frac{L}{L-1}} \mathbf{W}_{L} \boldsymbol{b}_{0}=\sqrt{\frac{L}{L-1}} \operatorname{clr}\left(\boldsymbol{a}_{0}\right),
\end{aligned}
$$




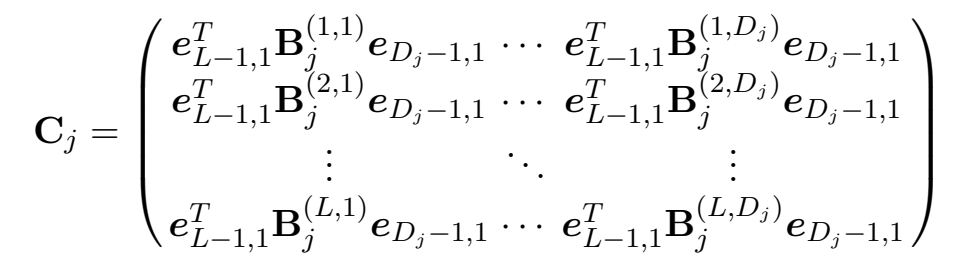

$$
\begin{aligned}
& =\left(\begin{array}{c}
\boldsymbol{e}_{L-1,1}^{T} \mathbf{W}_{L}^{T} \mathbf{P}_{L, 1} \mathbf{W}_{L} \mathbf{B}_{j} \mathbf{W}_{D_{j}}^{T}\left(\mathbf{P}_{D_{j}, 1}^{T} \mathbf{W}_{D_{j}} \boldsymbol{e}_{D_{j}-1,1}, \cdots, \mathbf{P}_{D_{j}, D_{j}}^{T} \mathbf{W}_{D_{j}} \boldsymbol{e}_{D_{j}-1,1}\right) \\
\boldsymbol{e}_{L-1,1}^{T} \mathbf{W}_{L}^{T} \mathbf{P}_{L, 2} \mathbf{W}_{L} \mathbf{B}_{j} \mathbf{W}_{D_{j}}^{T}\left(\mathbf{P}_{D_{j}, 1}^{T} \mathbf{W}_{D_{j}} \boldsymbol{e}_{D_{j}-1,1}, \cdots, \mathbf{P}_{D_{j}, D_{j}}^{T} \mathbf{W}_{D_{j}} \boldsymbol{e}_{D_{j}-1,1}\right) \\
\vdots \\
\boldsymbol{e}_{L-1,1}^{T} \mathbf{W}_{L}^{T} \mathbf{P}_{L, L} \mathbf{W}_{L} \mathbf{B}_{j} \mathbf{W}_{D_{j}}^{T}\left(\mathbf{P}_{D_{j}, 1}^{T} \mathbf{W}_{D_{j}} \boldsymbol{e}_{D_{j}-1,1}, \cdots, \mathbf{P}_{D_{j}, D_{j}}^{T} \mathbf{W}_{D_{j}} \boldsymbol{e}_{D_{j}-1,1}\right)
\end{array}\right) \\
& =\left(\begin{array}{c}
\boldsymbol{e}_{L-1,1}^{T} \mathbf{W}_{L}^{T} \mathbf{P}_{L, 1} \mathbf{W}_{L} \mathbf{B}_{j} \mathbf{W}_{D_{j}}^{T} \sqrt{\frac{D_{j}}{D_{j}-1}} \mathbf{G}_{D_{j}} \\
\boldsymbol{e}_{L-1,1}^{T} \mathbf{W}_{L}^{T} \mathbf{P}_{L, 2} \mathbf{W}_{L} \mathbf{B}_{j} \mathbf{W}_{D_{j}}^{T} \sqrt{\frac{D_{j}}{D_{j}-1}} \mathbf{G}_{D_{j}} \\
\vdots \\
\boldsymbol{e}_{L-1,1}^{T} \mathbf{W}_{L}^{T} \mathbf{P}_{L, L} \mathbf{W}_{L} \mathbf{B}_{j} \mathbf{W}_{D_{j}}^{T} \sqrt{\frac{D_{j}}{D_{j}-1}} \mathbf{G}_{D_{j}}
\end{array}\right) \\
& =\sqrt{\frac{D_{j}}{D_{j}-1}}\left(\begin{array}{c}
\boldsymbol{e}_{L-1,1}^{T} \mathbf{W}_{L}^{T} \mathbf{P}_{L, 1} \\
\boldsymbol{e}_{L-1,1}^{T} \mathbf{W}_{L}^{T} \mathbf{P}_{L, 2} \\
\vdots \\
\boldsymbol{e}_{L-1,1}^{T} \mathbf{W}_{L}^{T} \mathbf{P}_{L, L}
\end{array}\right) \mathbf{W}_{L} \mathbf{B}_{j} \mathbf{W}_{D_{j}}^{T} \mathbf{G}_{D_{j}} \\
& =\sqrt{\frac{D_{j}}{D_{j}-1}} \sqrt{\frac{L}{L-1}} \mathbf{G}_{L} \mathbf{W}_{L} \mathbf{B}_{j} \mathbf{W}_{D_{j}}^{T} \mathbf{G}_{D_{j}} \\
& =\sqrt{\frac{D_{j}}{D_{j}-1}} \sqrt{\frac{L}{L-1}} \mathbf{W}_{L} \mathbf{B}_{j} \mathbf{W}_{D_{j}}^{T}=\sqrt{\frac{D_{j}}{D_{j}-1}} \sqrt{\frac{L}{L-1}} \mathbf{A}_{j} .
\end{aligned}
$$

Therefore, $\boldsymbol{a}_{0}=\operatorname{clr}^{-1}\left(\sqrt{\frac{L-1}{L}} \boldsymbol{c}_{0}\right), \quad \mathbf{A}_{j}=\sqrt{\frac{L-1}{L}} \sqrt{\frac{D_{j}-1}{D_{j}}} \mathbf{C}_{j}(j=1,2, \cdots, q)$. 